\title{
POLYETHYLENE GLYCOL-400 USED AS PHASE TRANSFER CATALYST FOR ONE-POT SYNTHESIS OF 2-AMINO-3- CYANOPYRIDINE DERIVATIVES UNDER AQUEOUS CONDITIONS
}

\author{
Puligilla Balaswamy, Seema Aravind and Battu Satyanarayana* \\ Department of Chemistry, University College of Science, Osmania University, \\ Hyderabad, Telangana, India, 500007. \\ *E-mail: satyambchem@yahoo.co.in
}

\begin{abstract}
A green, multicomponent reaction method for the synthesis of 2-amino-3-cyanopyridine derivatives is developed by using Poly Ethylene Glycol (PEG-400) as phase transfer catalyst under aqueous conditions. Utilizing this protocol, 2-amino-3-cyanopyridines (5a-l) are synthesized by condensation followed by reactions of benzaldehyde, malononitrile, acetophenone and ammonium acetate. The prominent points for the present methodology are the use of a green solvent, cleaner reaction profile, efficiency, simplicity, easy work-up, excellent yields and recyclability of the solvent and final agreement with green chemistry protocols.

Keywords: PEG-400, Phase transfer catalyst, 2-amino-3-cyanopyridines, aqueous condition.
\end{abstract}

(C) RASĀYAN. All rights reserved

\section{INTRODUCTION}

The discovery of new methodologies for the synthesis of Nitrogen heterocyclics are thrust area in the field of organic, bioorganic and medicinal chemistry. One approach to fulfill this challenge involves the development of one-pot multicomponent reactions. The multicomponent reactions (MCRs) are prominent as an efficient and powerful technique in modern synthetic organic chemistry due to their important features. ${ }^{1-3}$ MCRs are an important in the synthesis of interesting heterocyclic scaffolds, particularly in complex molecules such as natural products and many other drugs. ${ }^{4}$ Pyridine and pyridine derivatives are heterocyclic compounds which are present in many natural and medicinal compounds. Therefore, the synthesis of pyridine derivatives is important in heterocyclic chemistry. ${ }^{5,6}$ This motivated us to synthesize 2-amino-3-cyano pyridines in the presence of PEG-400 as reaction medium and works as phase transfer catalyst (PTC) under aqueous conditions. 2-amino-3-cyano pyridine derivatives have attracted considerable interest as bioactive agents, ${ }^{7,8}$ such as A2A Adenosine receptor antagonists, ${ }^{9}$ IKK- $\beta$ inhibitors, ${ }^{10}$ a potent inhibitors of HIV-1 integrase, ${ }^{11}$ modulate androgen receptor function ${ }^{12}$ and anticancer agents. ${ }^{13}$ The focal point of research in organic chemistry is to investigate newer recyclable solvents, catalysts and environmentally friendly approaches. Various eco-hardships can be satisfied by using green catalyst and green solvents. ${ }^{14}$ The prime focus of our concern in the selection of solvent and catalyst was their unique properties such as inexpensive, immiscible with organic solvents and biodegradable water and PEG-400 as phase transfer catalyst. ${ }^{15-19}$ PEG-400 emerged as a powerful phase transfer catalyst and also a reaction medium for many organic transformations under mild reaction conditions. Moreover, PEG-400 is inexpensive, easy to handle, thermally stable, non-toxic and recyclable in various organic transformations, such as synthesis of thiadiazoles, ${ }^{20}$ quinazolines, ${ }^{21} \alpha$-amino acids ${ }^{22}$ and many transformations. ${ }^{23,24}$

\section{EXPERIMENTAL}

Materials and methods

Pre-coated silica-gel plates (E. Merck-60 $\mathrm{F}_{254}, 0.2 \mathrm{~mm}$ layer) for TLC and Column chromatography packed with Silica gel (60-120 mesh) were used. Fischer-John apparatus was used for melting point 
detection. Thermo Nicolet Nexus 670 FT-IR spectrometer was used to record IR spectrum. ${ }^{1} \mathrm{H}$ NMR and ${ }^{13} \mathrm{C}$ NMR spectra were recorded on Bruker-Avance $400 \mathrm{MHz}$ and $75 \mathrm{MHz}$ instruments using $\mathrm{CDCl}_{3}$ and TMS were used as internal standard. ESI-HRMS spectra recorded on Finnigan MAT 1020 mass spectrometer.

\section{General Procedure for synthesis of the 2-amino-3-cyano pyridine derivatives}

In a $50 \mathrm{ml}$ round bottom flask, aldehyde $(1.0 \mathrm{mmol})$, malononitrile $(1.0 \mathrm{mmol})$, acetophenone $(1.0 \mathrm{mmol})$ and ammonium acetate $(1.0 \mathrm{mmol})$ were dissolved in $30 \%(\mathrm{v} / \mathrm{v})$ polyethylene glycol in water, stirred with heating at $80^{\circ} \mathrm{C}$ for the $6 \mathrm{~h}$. The reaction progress was monitored by TLC. After completion of the reaction, the reaction mass was extracted with ethyl acetate. The organic portion was removed under reduced pressure and crude was purified by column chromatography. The PEG-400 was recovered and reused for four cycles without loss of significant activity.

\section{Spectral data of representative examples}

2-Amino-4, 6- diphenylnicotinonitrile (5a): Yield: 85\%; White solid; m.p. 181-183 ${ }^{\circ} \mathrm{C}$; ${ }^{1} \mathrm{H}$ NMR $\left(400 \mathrm{MHz}, \mathrm{CDCl}_{3}, \mathrm{ppm}\right): \delta 8.10(\mathrm{~s}, 1 \mathrm{H}), 7.48-7.20(\mathrm{~m}, 6 \mathrm{H}), 6.90(\mathrm{~s}, 7 \mathrm{H}), 6.83(\mathrm{~s}, 1 \mathrm{H}), 5.45(\mathrm{~s}, 2 \mathrm{H})$; ${ }^{13} \mathrm{CNMR}\left(75 \mathrm{MHz} \mathrm{CDCl}_{3}, \mathrm{ppm}\right): 155,154.66,147.32,147,139,136.23,132.23,129,128,126,124,118$, 111, 98, 77, 76; FT-IR (KBr, cm $\left.{ }^{-1}\right): 3129,2991,2914,2264,1751,1627,1573,1476,1403,1342,1243$, 1233, 1178, 1102, 1037, 978, 747, 522; ESI-HRMS [M+H] ${ }^{+} m / z$ Found: 271.1156. $\mathrm{C}_{18} \mathrm{H}_{13} \mathrm{~N}_{3}$ Calculated: 271.1142 .

2-Amino-4-(4-hydroxyphenyl) 6-phenyl nicotinonitrile (5b): Yield: $80 \%$; White solid; m.p. 226-228 ${ }^{\circ}$; ${ }^{1} \mathrm{HNMR}\left(400 \mathrm{MHz}, \mathrm{CDCl}_{3}\right.$, ppm) $\delta$ 8.10-7.90 (m, 3H). $7.75(\mathrm{~d}, 1 \mathrm{H}), 761-7.39(\mathrm{~m}, 7 \mathrm{H}), 5.40(\mathrm{~s}, 1 \mathrm{H}, \mathrm{OH})$; ${ }^{13} \mathrm{CNMR}\left(75 \mathrm{MHz} \mathrm{CDCl}_{3}, \mathrm{ppm}\right): 164.52,164,158.23,158,141,139.11,134.21,133,132,130,120,114$, 106, 80; FT-IR $\left(\mathrm{KBr}, \mathrm{cm}^{-1}\right): 3389,3168,3056,2264,1723,1620,1598,1540,1481,1340,1245,1098$, 842, 741, 610, 551; ESI-HRMS [M+H] ${ }^{+} m / z$ : Found: 287.1148, $\mathrm{C}_{18} \mathrm{H}_{13} \mathrm{~N}_{3} \mathrm{O}$, Calculated: 287.1156.

2-Amino-4-(4-bromophenyl) 6-phenyl nicotinonitrile (5c): Yield: 82\%; Yellow solid; m.p. 191-193 C; ${ }^{1} \mathrm{HNMR}\left(400 \mathrm{MHz}, \mathrm{CDCl}_{3}, \mathrm{ppm}\right) \delta 8.20(\mathrm{~d}, 2 \mathrm{H}), 8.01(\mathrm{~s}, 1 \mathrm{H}), 7.71-7.30(\mathrm{~m}, 10 \mathrm{H}) ;{ }^{13} \mathrm{CNMR}(75 \mathrm{MHz}$ $\left.\mathrm{CDCl}_{3}, \mathrm{ppm}\right): 165,159,143,141,137,135,134,133,132,129,121,115,92,81$; FT-IR $\left(\mathrm{KBr}, \mathrm{cm}^{-1}\right)$ : $3448,2924,2853,2243,1613,1578,1508,1495,1458,1381,1265,755,575$; ESI-HRMS $[\mathrm{M}+\mathrm{H}]^{+} m / z$ : Found : $350.2156, \mathrm{C}_{18} \mathrm{H}_{12} \mathrm{BrN}_{3}$ Calculated: 349.0216 .

2-Amino 6-phenyl -4-p-tolylnicotinonitrile (5d): Yield: 85\%; White solid; m.p. 170-173 ${ }^{\circ} \mathrm{C}$; ${ }^{1} \mathrm{HNMR}$ $\left(400 \mathrm{MHz}, \mathrm{CDCl}_{3}, \mathrm{ppm}\right) \delta$ 7.70-7.27 (m, 10H), $4.15(\mathrm{~m}, 1 \mathrm{H}), 3.85(\mathrm{~m}, 1 \mathrm{H}), 1.25(\mathrm{~m}, 3 \mathrm{H}) ;{ }^{13} \mathrm{C} \mathrm{NMR}$ (75MHz $\left.\mathrm{CDCl}_{3}, \mathrm{ppm}\right): 159,154,153,150,139,137,135,132,131,129,125,119,113,105,89,79,36$; FT-IR (KBr, cm $\left.{ }^{-1}\right)$ : 3462, 3305, 2967, 2924, 2832, 2203, 1751, 1638, 1577, 1509, 1452, 1368, 1296, 1250, 1176, 1109, 1025, 821, 767, 701, 520, ESI-HRMS $[\mathrm{M}+\mathrm{H}]^{+} m / z$ Found: $285.1386, \mathrm{C}_{19} \mathrm{H}_{15} \mathrm{~N}_{3}$ Calculated: 285.1362.

2-Amino -4-(naphthalene1-yl)6-phenyl nicotinonitrile (5e): Yield: 75\%; White solid; m.p. 175-177 ${ }^{\circ}$; ${ }^{1} \mathrm{HNMR}\left(400 \mathrm{MHz}, \mathrm{CDCl}_{3}\right.$, ppm) $\delta 8.75(\mathrm{~s}, 1 \mathrm{H}), 8.21-8.17(\mathrm{~m}, 3 \mathrm{H}), 7.70(\mathrm{~s}, 2 \mathrm{H}), 7.56-7.22(\mathrm{~m}, 6 \mathrm{H}), 6.90$ $(\mathrm{s}, 1 \mathrm{H}), 6.78(\mathrm{~s}, 1 \mathrm{H}), 6.31(\mathrm{~s}, 1 \mathrm{H}) ;{ }^{13} \mathrm{CNMR}\left(75 \mathrm{MHz}, \mathrm{CDCl}_{3}, \mathrm{ppm}\right): 159.15,158.96,154,147,144.62$, $144,143,142,141,136,135,131,129,128.5,128,127,126,123,109,96,90$; FT-IR $\left(\mathrm{KBr}, \mathrm{cm}^{-1}\right)$ : 3463, 3129, 2991, 2814, 2204, 1751, 1627, 1616, 1452, 1402, 1342, 1249, 1176, 1102, 1037, 978, 747, 522,; ESI-HRMS [M+H] $]^{+} m / z$ : Found: 321.1365. $\mathrm{C}_{22} \mathrm{H}_{15} \mathrm{~N}_{3}$. Calculated: 321.1316.

2-Amino-4-(4-methoxyphenyl)-6-phenylnicotinonitrile (5f): Yield: 78\%; White solid; m.p. 178-180 ${ }^{\circ}$; ${ }^{1} \mathrm{H}$ NMR $\left(400 \mathrm{MHz}, \mathrm{CDCl}_{3}, \mathrm{ppm}\right) \delta 8.10(\mathrm{~d}, 1 \mathrm{H}), 7.64-7.31(\mathrm{~m}, 8 \mathrm{H}), 5.42(\mathrm{~s}, 1 \mathrm{H}), 2.15(\mathrm{~s}, 3 \mathrm{H}) ;{ }^{13} \mathrm{C} \mathrm{NMR}$ (75MHz, $\left.\mathrm{CDCl}_{3}, \mathrm{ppm}\right): 163,162.5,162,161.5,160,154,139,131.5,131,130.5,130,129,128,114,111$, 77, 76.5; FT-IR (KBr, cm-1): 3463, 3306, 3182, 2967, 2924, 2632, 2204, 1639, 1577, 1509, 1452, 1215, 
RASĀYAN $J$. Chem.

Vol. 10 | No. 4 |1334-1339 | October - December | 2017

1179, 1109, 1025, 929, 767, 620; ESI-HRMS [M+H] $]^{+} m / z$ : Found: 301.9822; $\mathrm{C}_{19} \mathrm{H}_{15} \mathrm{~N}_{3} \mathrm{O}$ Calculated: 301.1224 .

2-Amino-6-phenyl-4-(thiophen-2-yl) nicotinonitrile (5g): Yield: 80\%; White solid; m.p. 160-163 ${ }^{\circ}$; ${ }^{1} \mathrm{H}$ NMR $\left(400 \mathrm{MHz}, \mathrm{CDCl}_{3}\right.$, ppm) $\delta$ 8.06-7.99 (m, 1H), 7.17-7.07 (m, 10H), $6.98(\mathrm{~s}, 1 \mathrm{H}) ;{ }^{13} \mathrm{C}$ NMR $(75 \mathrm{MHz}$, $\left.\mathrm{CDCl}_{3}, \mathrm{ppm}\right): 160,148,144,141,129,128,127,121,118,113,112,106,82$, 77, 76.5, 76; FT-IR (KBr, $\left.\mathrm{cm}^{-1}\right): 3390,3169,3056,2264,1710,1504,1489,1381,1245,1065,841,748$; ESI-HRMS [M+H] ${ }^{+} m / z$ : Found: 277.0742; $\mathrm{C}_{16} \mathrm{H}_{11} \mathrm{~N}_{3} \mathrm{~S}$, Calculated: 277.0713.

2-Amino-4-(furan-2-yl)-6-phenylnicotinonitrile (5h): Yield: $78 \%$; Yellow solid; m.p. $155-157^{\circ} \mathrm{C}$; ${ }^{1} \mathrm{H}$ NMR (400MHz, $\mathrm{CDCl}_{3}$, ppm) $\delta 7.96(\mathrm{~s}, 1 \mathrm{H}), 7.70-7.57(\mathrm{~m}, 4 \mathrm{H}), 7.50-7.27(\mathrm{~d}, 2 \mathrm{H}), 7.25-6.85(\mathrm{~m}, 4 \mathrm{H})$; ${ }^{13} \mathrm{C}$ NMR (75MHz, $\left.\mathrm{CDCl}_{3}, \mathrm{ppm}\right): 162,152,147,144,141,133,132,131,120,116,115,109,96.5,96$, 91.5, 91; FT-IR (KBr, cm$\left.{ }^{-1}\right): 3390,3169,3056,2264,1710,1504,1489,1381,1265,1245,1101,1065$, 841, 748; ESI-HRMS [M+H] $]^{+} m / z$ : Found: 261.1012, $\mathrm{C}_{16} \mathrm{H}_{11} \mathrm{~N}_{3} \mathrm{O}$, Calculated: 261.0954;

2-Amino-4-(4-nitrophenyl)-6-phenylnicotinonitrile (5i): Yield: 88\%; Yellow liquid; b.p. 271-273 ${ }^{\circ}$; ${ }^{1} \mathrm{H}$ NMR $\left(400 \mathrm{MHz}, \mathrm{CDCl}_{3}, \mathrm{ppm}\right) \delta 7.60-7.23(\mathrm{~m}, 8 \mathrm{H}), 6.99-6.75(\mathrm{~m}, 3 \mathrm{H}), 6.10(\mathrm{~s}, 1 \mathrm{H}) ;{ }^{13} \mathrm{C} \mathrm{NMR}$ $\left(75 \mathrm{MHz}, \mathrm{CDCl}_{3}, \mathrm{ppm}\right): 165,164.9,159,144,141,137,133,132,129,121,116.5,116,92,82,81.5$, 81;FT-IR(KBr, $\left.\mathrm{cm}^{-1}\right)$ : 3462, 3308, 3182, 2967, 2924, 2832, 2209, 1639, 1577, 1509, 1452, 1250, 1171, 1025, 821, 707, 620; ESI-HRMS [M+H] ${ }^{+} m / z$ : Found: $317.2154 \mathrm{C}_{18} \mathrm{H}_{12} \mathrm{~N}_{4} \mathrm{O}_{2}$, Calculated: 316.1092.

2-Amino-6-phenyl-4(1H-pyrrol-2-yl) nicotinonitrile (5j): Yield: 75\%; White solid; m.p. 182-184 ${ }^{\circ} \mathrm{C}$; ${ }^{1} \mathrm{HNMR}\left(400 \mathrm{MHz}, \mathrm{CDCl}_{3}\right.$, ppm) $\delta$ 8.12-7.92 (s, $\left.1 \mathrm{H}\right), 7.68-7.45(\mathrm{~m}, 5 \mathrm{H}), 7.42-7.29(\mathrm{~m}, 3 \mathrm{H}), 2.58-2.29(\mathrm{~m}$, $3 \mathrm{H}, \mathrm{NH}, \mathrm{NH} 2) ;{ }^{13} \mathrm{C} \mathrm{NMR}\left(75 \mathrm{MHz}, \mathrm{CDCl}_{3}, \mathrm{ppm}\right): 159,158,148,144,141,134,129.5,129,128,127$, 121, 113, 112, 106, 82, 76.5, 76; FT-IR $\left(\mathrm{KBr}, \mathrm{cm}^{-1}\right): 3389,3168,3056,2264,1723,1621,1598,1540$, 1481, 1340, 1245, 1098, 842, 741, 610, 551; ESI-HRMS $[\mathrm{M}+\mathrm{H}]^{+} m / z$ : Found: $261.1146, \mathrm{C}_{16} \mathrm{H}_{12} \mathrm{~N}_{4}$, Calculated: 260.1116.

2-Amino-4-(4-chlorophenyl)-6-phenylnicotinonitrile (5k): Yield: 83\%; White solid; m.p. 227-229 C; ${ }^{1} \mathrm{HNMR}\left(400 \mathrm{MHz}, \mathrm{CDCl}_{3}, \mathrm{ppm}\right) \delta$ 7.99 (s, 1H), 7.93-7.87 (d, 2H, NH2), 7.59-7.35 (m, 7H), 7.34-7.26 (t, 1H), 7.23-7.14 (t, 1H); ${ }^{13} \mathrm{C}$ NMR $\left(75 \mathrm{MHz}, \mathrm{CDCl}_{3}, \mathrm{ppm}\right): 165,158,142,141,139,135,134,133,132$, 131, 116, 115, 91, 81; FT-IR (KBr, cm $\left.{ }^{-1}\right): 3463,3129,2991,2914,2203,17512,1642,1587,1465,1342$, 1243, 1178, 10337, 747, 522; ESI-HRMS $[\mathrm{M}+\mathrm{H}]^{+} m / z$ : Found: 305.0712, $\mathrm{C}_{18} \mathrm{H}_{12} \mathrm{ClN}_{3}$, Calculated: 305.0762 .

2' -Amino-6' -phenyl-2, 4' -bipyridine-3' -carbonitrile (5l): Yield: 80\%; White solid; m.p. 211-213 ${ }^{\circ} \mathrm{C}$; ${ }^{1} \mathrm{H}$ NMR (400 MHz, $\left.\mathrm{CDCl}_{3}, \mathrm{ppm}\right) \delta 8.10(\mathrm{~m}, 3 \mathrm{H}), 7.70(\mathrm{~s}, 1 \mathrm{H}), 7.59-7.22(\mathrm{~m}, 6 \mathrm{H}), 6.88(\mathrm{~s}, 1 \mathrm{H}), 6.78(\mathrm{~s}, 1 \mathrm{H})$; ${ }^{13} \mathrm{C}$ NMR (75MHz, $\left.\mathrm{CDCl}_{3}, \mathrm{ppm}\right): 162,155,154,151,139,137,135,132,131,129,126,118,112,89$, 78.5, 78, 77.5; FT-IR (KBr, cm $\left.{ }^{-1}\right): 3462,3389,3145,2964,2261,1723,1620,1540,1451,1340,1248$, 1098, 842, 741; ESI-HRMS [M+H] $]^{+} m / z$ : Found: 272.2158; $\mathrm{C}_{17} \mathrm{H}_{12} \mathrm{~N}_{4}$, Calculated: 272.1118 .

\section{RESULTS AND DISCUSSION}

Employing the reaction using various aromatic aldehydes, malononitrile, acetophenone, ammonium acetate in presence of PEG-400 and aqueous media refluxed for $6 \mathrm{~h}$, obtained the products in excellent yields (Scheme-1).

\section{Optimization}

We studied different methods for synthesis of 2-amino-3-cyano pyridine derivatives, in various solvents and catalysts at different proportion conditions. It was observed that trace amount of product (10\% yield) formed in solvent-free and aqueous conditions (Entry 1 in Table. 1). In organic solvents such as DCM and DMF, media product was afforded in 30, 35\% yield after 6 h reaction (Entry 2 and 3 in Table. 1). A 
moderate yield was obtained in polyethylene glycol and good yield was observed in PEG-400, water mixtures at $80{ }^{\circ} \mathrm{C}$ (Entry 4, 5, 6 and 7 in Table.-1). However, it was found that 30\% of PEG-400 in water to be an essential promoter for this reaction, as can be seen from the Table-1. It seems that PEG-400 in water at different proportions given a different range of yields.

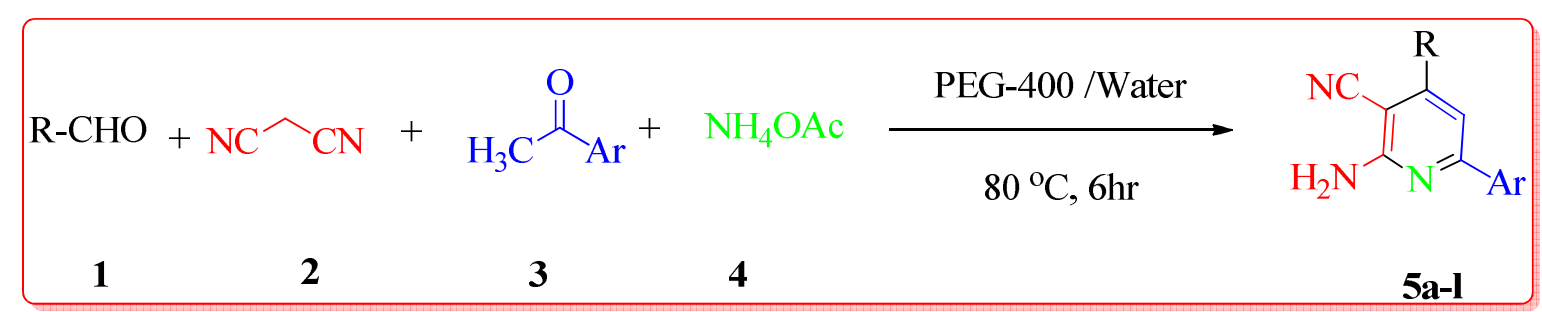

Scheme-1

Table-1: Optimization of reaction conditions.

\begin{tabular}{l|l|l|l|l}
\hline Entry & Solvent & Temp. ${ }^{\circ} \mathrm{C}$ & Time (h) & Yield (\%) \\
\hline 1. & Neat/Water & 80 & 6 & 10 \\
\hline 2. & DCM & 80 & 6 & 30 \\
\hline 3. & DMF & 80 & 6 & 35 \\
\hline 4. & PEG-400 & 80 & 6 & 40 \\
\hline 5. & PEG (10\%) in Water & 80 & 6 & 70 \\
\hline 6. & PEG (20\%) in Water & 80 & 6 & 80 \\
\hline 7. & PEG (30\%) in Water & 80 & 6 & 85 \\
\hline 8. & PEG (50\%) in water & 80 & 6 & 75 \\
\hline
\end{tabular}

The PEG-400 in water acts as PTC which promotes the reaction by interfering with reactants and facilitates to transfer from one phase to another phase of all intermediates in the reaction. The reaction mechanism involves addition followed by aromatization through the enamine intermediate formation, which is shown in Fig.-1.The phase transfer catalyst mechanism is shown in Fig.-2.

PEG-400 was recollected from the reaction mixture and reused four with same reaction conditions for four to five times and observed that PEG-400 has shown good phase transfer catalytic activity with 5\% loss of its activity.

Table-2: Recycling of PEG-400/ $\mathrm{H}_{2} \mathrm{O}$.

\begin{tabular}{l|l|l|l|l|l}
\hline No. of Cycles & Fresh & Run1 & Run2 & Run3 & Run4 \\
\hline Yield(\%) & 80 & 80 & 79 & 78 & 77 \\
\hline Time (min) & 120 & 120 & 120 & 120 & 120 \\
\hline
\end{tabular}

The reaction was established by synthesis of various 2-Amino-3-cyano pyridine derivatives using simple starting materials. In general, all the obtained products were clean and structures were confirmed by ${ }^{1} \mathrm{H}$ NMR, ${ }^{13} \mathrm{C}$ NMR, IR and Mass Spectral Analysis.

Table-3: Synthesis of various 2-Amino-3-Cyanopyridine derivatives by MCR.

\begin{tabular}{|c|c|c|c|c|}
\hline Entry & R-CHO & $\begin{array}{l}\mathrm{ArCO} \\
\mathrm{CH}_{3}\end{array}$ & Time (h) & Yield (\%) \\
\hline $5 a$ & $\mathrm{Ph}-$ & Ph- & 6 & 85 \\
\hline $5 b$ & 4-OH- Ph- & Ph- & 6 & 80 \\
\hline $5 c$ & 4-Br- $\mathrm{Ph}-$ & Ph- & 6 & 82 \\
\hline $5 \mathrm{~d}$ & $4-\mathrm{CH}_{3}-\mathrm{Ph}-$ & Ph- & 6 & 85 \\
\hline $5 e$ & 1-Naphtha- & Ph- & 6 & 75 \\
\hline $5 f$ & $4-\mathrm{OCH}_{3}-\mathrm{Ph}-$ & Ph- & 6 & 78 \\
\hline $5 g$ & Thiophene-2- & Ph- & 6 & 75 \\
\hline
\end{tabular}




\begin{tabular}{l|l|l|l|l}
\hline $5 \mathrm{~h}$ & Furan-2- & $\mathrm{Ph}-$ & 6 & 80 \\
\hline $5 \mathrm{i}$ & 4-NO ${ }_{2}-\mathrm{Ph}-$ & $\mathrm{Ph}-$ & 6 & 86 \\
\hline $5 \mathrm{j}$ & Pyrrolo-2- & Ph- & 6 & 75 \\
\hline $5 \mathrm{k}$ & 4-Cl-Ph- & Ph- & 6 & 83 \\
\hline $5 \mathrm{l}$ & Pyridine-2- & Ph- & 6 & 80 \\
\hline
\end{tabular}

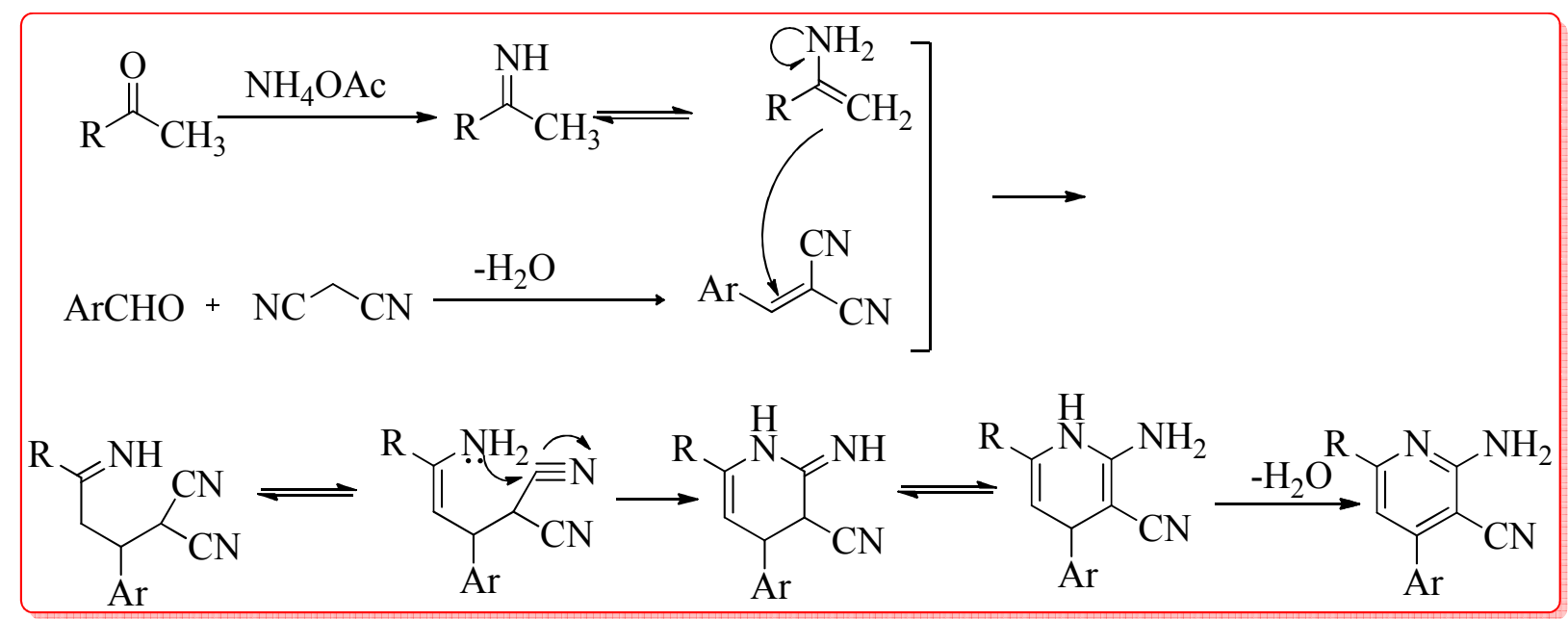

Fig.-1: Reaction mechanism

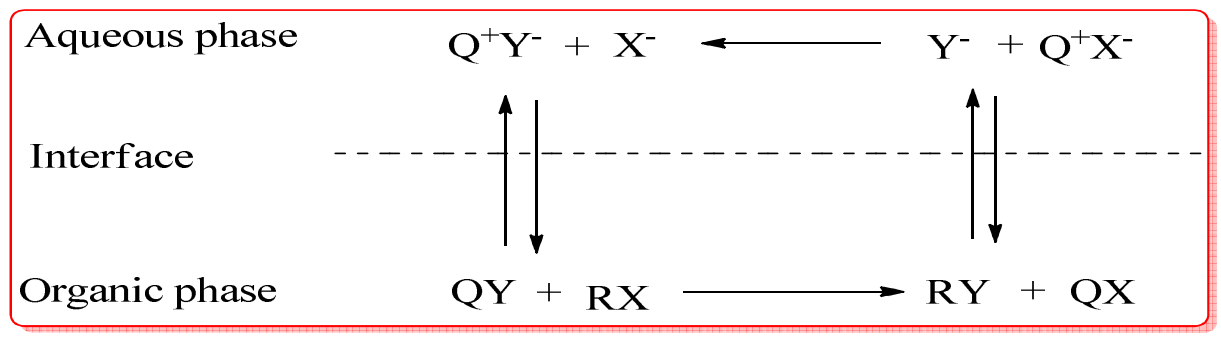

Fig.-2: Phase transfer catalyst mechanism

\section{CONCLUSION}

In summary, we have developed an efficient and facile method to synthesize 2-Amino-3-cyanopyridine derivatives using aldehyde, acetophenone, malononitrile and ammonium acetate in aqueous medium and PEG-400 as phase transfer catalyst. The advantage of this method is utilizing of green chemistry protocols such as multicomponent reaction with high yields and mild reaction conditions. PEG-400 was used as PTC and reaction medium in various reactions, it may be applicable for development of new synthetic methods.

\section{ACKNOWLEDGEMENT}

The authors are thankful to Department of Chemistry, Osmania University, Hyderabad, India-500007, for constant encouragement, generously supporting and providing facilitation in this research work as well as financial support.

\section{REFERENCES}

1. D. J. Ramon and Y. Miguel, Angew. Chem. Int. Ed., 44, 1602 (2005).

2. R.V. A. Orru and M. de Greef, Synthesis., 10, 1471 (2003).

3. I. Ugi and S. Heck, Comb. Chem. High Throughput Screen., 4, 1 (2001).

4. B. E. Evans, J. Med. Chem., 31, 2235 (1988).

5. S. N. Dattatray, V. C. Hemant, B. D. Sakharam and B. H. Vasant, Synth. Commun., 46, 1665 (2016).

6. R. A. Bawa and A. M. Beatty, Rasayan. J. Chem., 5(4), 496 (2012). 
RASĀYAN J. Chem.

Vol. 10 | No. 4 |1334-1339 | October - December | 2017

7. S. Drabu, Archna, S. Singh, S. Munirajam and N. Kumar, Indian J. Heterocycl. Chem., 16, 411 (2007).

8. P. Victory, J. Cirujeda and A. Anton Vidal-Ferran, Tetrahedron., 51, 10253 (1995).

9. B. C. H. May, J. A. Zorn, J. Witkop, J. Sherrill, A. C. Wallace, G. Legname, S. B. Prusiner and F.E. Cohen, J. Med. Chem.,50, 65 (2007).

10. M. Mantri, O. De Graaf, J. Van Veldhoven, A. Goblyos, J. K. Von Frijtag Drabbe Kunzel, T. Mulder-Krieger, R. Link, H. De Vries, M. W. Beukers, J. Brussee and A. P. Ijzerman, J. Med. Chem.,51, 4449 (2008).

11. T. Murata, M. Shimada, S. Sakakibara, T. Yoshino, H. Kadono, T. Masuda, M. Shimazaki, T. Shintani, K. Fuchikami, K. Sakai, H. Inbe, K. Takeshita, T. Niki, M. Umeda, K.B. Bacon, K. B. Ziegelbauer and T.B. Lowinger, Bioorg. Med. Chem. Lett., 13, 913 (2003).

12. J. Deng, T. Sanchez, L. Q. Al-Mawsawi, R. Dayam, R. A. Yunes, A. Garofalo, M. B. Bolger and N. Neamati, Bioorg. Med. Chem.,15, 4985 (2007).

13. A. S. Girgis, A. Kalmouch and H. M. Hosni, Amino Acids., 26, 139 (2004)

14. P. Balaswamy, S. Aravind, B. Satyanarayana, Rasayan. J. Chem. 10(1), 6 (2017).

15. A. E. D1'az-A' lvarez, J. Francos, B. Lastra-Barreira, P. C. V. Cadierno. Chem. Commun., 47, 6208 (2011)

16. R. Neumann and Y. Saaaon, Tetrahedron., 39 (20), 3437(1983).

17. G. E. Totten and N. A. Clinton, J. Macromol. Sci. Part C. Polymer. Rev., 38(1), 77 (1998).

18. A. S. Shedge, B. P. Kavitake, U. V. Desai and P. P. Wadgaonkar, Synth. Commun., 34(24), 4483 (2004).

19. A. Djemilia, S. Lakrouta, Z. Cheraieta, M. Berredjema and N. E. Aoufa. Green Chem. Lett. Rev., 8, $48(2015)$.

20. X. Wang, Z. Li and J. Yang, Synth. Commun., 31(9), 1447 (2001).

21. P. Ramesh and K. Bhaskar, J. Chem. Pharm. Res., 5, 314 (2013).

22. B. Sauvagnat, K. Kulig, F. Lamaty, R. Lazaro and J. Martinez, J. Comb. Chem., 2(2), 134 (2000).

23. N. Suryakiran, T. Srikanth Reddy, K. Ashalatha, M. Lakshman and Y. Venkateswarlu, Tetrahedron Lett., 47, 3853 (2006).

24. Y. R. Jorapur, G. Rajagopal, P. J. Saikia and R. R. Pal, Tetrahedron Lett., 49(9), 1495 (2008).

[RJC-1903/2017] 